\title{
Performance Indicators Modern Surveillance Radar
}

\author{
Peter N.C. Nooij \\ Thales Nederland \\ Hengelo (Ov.) \\ Netherlands \\ peter.nooy@nl.thalesgroup.com
}

\author{
Arne Theil \\ Netherlands Organization of Applied Scientific Research TNO \\ The Hague \\ Netherlands \\ arne.theil@tno.nl
}

\begin{abstract}
Blake chart computations are widely employed to rank detection coverage capabilities of competitive search radar systems. Developed for comparable 2D radar systems with a mechanically rotating reflector antenna, it was not necessary to regard update rate and plot quality in Blake's chart. To characterize the system's critical awareness, the inclusion of update rate is, however, of utmost importance. Performance indicators that are considered in this communication therefore contain update rate, resulting in a coverage rate metric. Plot quality is integrated in the coverage information rate metric, which relates to the system's situational awareness as well. It is concluded that Blake chart-only comparison is not sufficient to rank competing modern search radar systems based on different radar principles. Coverage information rate is elaborated for several radar principles, in chronological order starting with Blake's rotating 2D search radar and finalizing with a modern non-rotating four face fixed phased array 4D surveillance radar.
\end{abstract}

Keywords-Blake chart; coverage; coverage rate ; $2 D-4 D$ resolution; coverage information rate

\section{INTRODUCTION}

Quick radar performance comparisons are dominated by coverage computations based on radar charts as introduced by Lamont V. Blake in 1962 [1] as shown in Fig. 1. The Blake chart has been developed for 2D search radar of the 1960's, applying comparable principles in terms of antenna, waveform, detection and plot extraction, i.e.:

- Antenna: Rotating reflector antenna with a $\operatorname{cosec}^{2}$ like elevation beam pattern.

- Waveform: Low-PRF (unambiguous range).

- Detection: Binary integration and 2D estimation of the target's range-bearing position by leading/ trailing edge over the clustered pre-detections in range and azimuth ("bit-map" with ones and zeroes).

Blake chart comparisons for radar look-a-likes resulted in straightforward ranking of operational 2D performance potencies. Dedicated to designate the "threat", search radar development was governed by the maximum range requirement. The $2 \mathrm{D}$ search radar target data was sent to the fire channel of the assigned effector to neutralize the threat. Operator designation was based on a "blip" on the plan position indicator (PPI), a range-bearing display.

\footnotetext{
${ }^{1}$ The manuscript of this paper was prepared in the framework of D-RACE (Dutch RAdar Centre of Expertise, a strategic alliance of Thales Nederland B.V. and TNO).
}

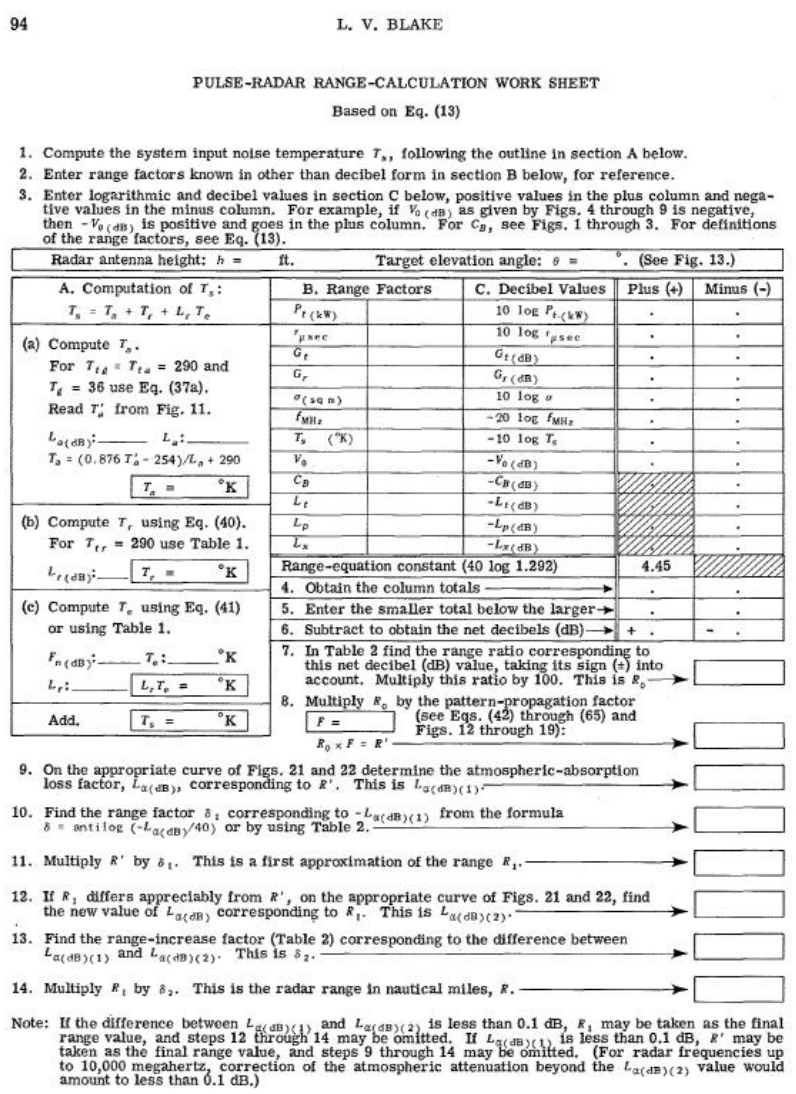

Fig. 1. Blake's chart to determine maximum radar detection range

Since that era, -some fifty years ago-, extensive changes have taken place in both operational missions and radar principles. A variety of complex operational warfare ${ }^{2}$ requirements ${ }^{3}$ are integrated and a multitude of different search radar principles are offered nowadays, i.e. 2D, 3D and "4D" (position plus radial velocity) with rotating and multiple fixed antenna panels or "faces". The enhanced angular radar resolution during the years of rotating search radar evolution is illustrated in Fig. 2.

Nowadays, the majority of military missions consists of peacekeeping operations. Consequently, search radar is subjected to support both critical and situational awareness

\footnotetext{
${ }^{2}$ E.g. littoral warfare, asymmetrical threat masked by or disguised as civil traffic

${ }^{3}$ E.g. automatic tracking, classification, identification of all radar targets of interest within the coverage
} 
tasks. Low false alarm rate multi-target tracking, target classification and identification (friend or foe) are important qualities, i.e. more important than long range detection of a potential threat. Hereto Blake's coverage alone is not sufficient. Blake's chart fails to take into account the track quality, which is determined by the dimension (2D, 3D, 4D) and resolution of the plot in the search volume covered. Alternative performance measures are introduced in this paper. The update rate of the search volume is introduced in the "Coverage Rate" (CR). The update rate and quality of the radar information is integrated in the "Coverage Information Rate" (CIR). The latter gives an indication of the potential track-, classification- and identification-quality within the coverage.

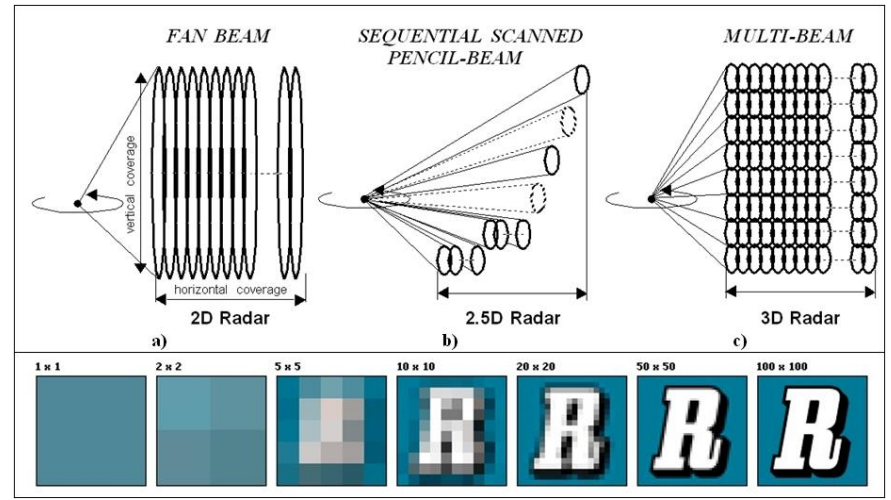

Fig. 2. Rotating antenna evolution going from Blake's a) fan beam, to b) elevation scanned pencil beam (sequential lobing), and c) multi-beam (simultaneous lobing). Modern multi-beam in azimuth and elevation is not shown. 4D radar refers to instantaneous radial velocity measurement with multi-beam

\section{PERFORMANCE INDICATORS}

\section{A. Coverage Rate}

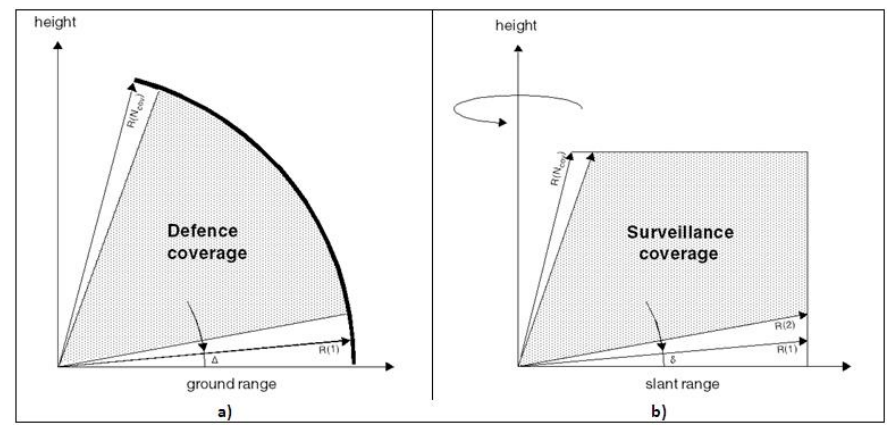

Fig. 3. Illustration of the covered search volume per time unit or coverage rate for a) $\mathrm{CR}_{\mathrm{el}}$ in $[\mathrm{km} . \% \mathrm{~s}]$ supporting critical awareness or Defence against diving missile threat between $0-70^{\circ}$ elevation, b) $\mathrm{CR}_{\mathrm{V}}$ in $\left[\mathrm{km}^{3} / \mathrm{s}\right]$ supporting situational awareness or Surveillance for constant level flight targets

Air Defence requires coverage as shown in Fig. 3a) to establish similar track-while-scan initiation or initial "designation" ranges in elevation. In this manner in-time neutralization of the diving missile threat, -e.g. between $0-70^{\circ}$ elevation can be achieved. Formula (1) can be derived for $\mathrm{m}=$ $1, \ldots, \mathrm{N}$ search volumes to estimate the integrated $\mathrm{CR}$.
Blake's range coverage ${ }^{4} \mathrm{R}_{\mathrm{m}}(\mathrm{i})$ at elevation $\theta_{\mathrm{el}}(\mathrm{i})=\mathrm{i} . \Delta(\Delta=$ elevation step in degree in $\left[\mathrm{E}_{\min }, \mathrm{E}_{\max }\right]$ domain) and update time $T_{m}$ of search volume $m$ are equally ${ }^{5}$ weighted

$$
C R_{e l} \cong \sum_{m=1}^{N} \frac{\Delta}{T_{m}}\left\{\sum_{i=1}^{N_{c o v}} R_{m}(i)\right\}
$$

Air Surveillance supports air traffic control and situational awareness tasks. Cosec $^{2}$-pattern type of coverage as shown in Fig. 3b) secures track continuity of constant level flight air traffic after initial detection. In this manner knowledge of the target (e.g. state, identification, classification) is maintained within the coverage because track loss is prevented. The volume coverage rate $\mathrm{CR}_{\mathrm{V}}$ is approximated by integration in elevation ( $\delta=$ elevation step in radians) over the azimuth coverage $\varphi_{\text {cov }}$ for the search volumes examined

$$
C R_{V} \cong \sum_{m=1}^{N} \frac{\varphi_{c o v}(m)}{3 T_{m}}\left\{\sum_{i=1}^{N_{c o v}} R_{m}^{3}(i) \cdot \delta \cdot \cos \left(\theta_{e l}(i)\right)\right\}
$$

\section{B. Coverage Information Rate}

CIR expands CR with the radar plot resolution. CIR is a measure for the appreciation of the surveillance radar during peace-time operations and in evaluating the asymmetric threat. Within Blake's coverage, CIR qualifies aspects such as plotresolution, -discrimination, -accuracy, track-continuity, classification, -identification, radar robustness and reliability (i.e. low false alarm and track rate). The following coverage ${ }^{6}$ and 4D radar effective ${ }^{7}$ resolution (indicated with $\Delta$ ) aspects per search volume are integrated in the $\mathrm{CR}_{\mathrm{el}}$ expression (1)

- Radial Range: Coverage within unambiguous range, i.e. $R(i)=\max \left(R_{\min }, \min \left(R_{\max }, R_{o}(i)\right)\right.$, resolution $\Delta R$.

- Radial Velocity: Two-sided unambiguous radial velocity: $\mathrm{N}_{\mathrm{Vrad}}=2\left(\mathrm{~V}_{\max }-\mathrm{V}_{\min }\right) / \Delta \mathrm{V}_{\mathrm{rad}}$.

- Azimuth: Required instrumented bearing coverage: $\mathrm{N}_{\mathrm{B}}=\left(\mathrm{B}_{\max }-\mathrm{B}_{\min }\right) / \Delta \mathrm{B}$.

- Elevation: Instantaneous resolution $\Delta \mathrm{E}$ within $\left[\mathrm{E}_{\mathrm{min}}\right.$, $\left.\mathrm{E}_{\max }\right]$, i.e. the elevation domain to be covered.

CIR in $\left[\mathrm{s}^{-1}\right]$ is defined here as

$$
C I R \cong \sum_{m=1}^{N} \frac{\Delta \cdot N_{B} \cdot N_{V_{\text {rad }}}}{\Delta R \cdot \Delta E \cdot T_{m}}\left\{\sum_{i=1}^{N_{c o v}} R_{m}(i)\right\}
$$

\footnotetext{
${ }^{4}$ Blake's chart result $\mathrm{R}_{\mathrm{o}}$ is the free space detection range for the target radar cross section, Swerling case and elevation of interest as computed in the Blake chart in Fig. 1

${ }^{5}$ Uniform weights for radar coordinates range, elevation and update time are used

${ }^{6}$ Instrumented or required coverage per radar coordinate is indicated by e.g. $R_{\min }, R_{\max }$

${ }^{7}$ Effective resolution is dependent on wave-, beam-form agility, radar resolution $-3 \mathrm{~dB}$ response width, sampling and number of coherent measurements to form a plot, e.g. $\Delta \mathrm{E}=$ effective elevation resolution
} 
This is an integrated measure of the plot/track quality, responsiveness and robustness within Blake's coverage, practically expressed in $[\mathrm{dB} / \mathrm{s}]$.

\section{SEARCH RADAR ANALYSIS}

\section{A. Defence: Multi-beam vs. Sequential Scanned Pencil Beam}

The reaction time, defined by the time from initial detection up to firm track is critical in many threat scenarios. "Firm" means that the track vector's accuracy (to predict its destination) and identification (friend/foe) are credible to start threat evaluation and weapon assignment. Sophisticated search radar simulators can produce firm track range statistics. These require detailed, mostly classified radar-, target- and scenariodata, as well as an up-to-date thoroughly validated radar model. CR computations are less detailed and provide a straightforward metric for the inherent search radar's coverage capability and responsiveness. Obviously, update-rate and related reaction-time are of great importance for close-in agile threat scenarios, such as a high speed maneuverable missile threat launched from platforms behind coastlines, submarines or surface vessels. To illustrate the added value of the CR metric a comparison is made for two 30 RPM radar systems: a) a Sequential Scanned Pencil Beam (25 E-scan positions) and b) a 3D Multi-beam (six simultaneous beams) search radar principle.

The Blake chart coverage is shown in Fig. 4. The conclusion is crystal clear: The Sequential Scanned Pencil Beam radar is superior in coverage.

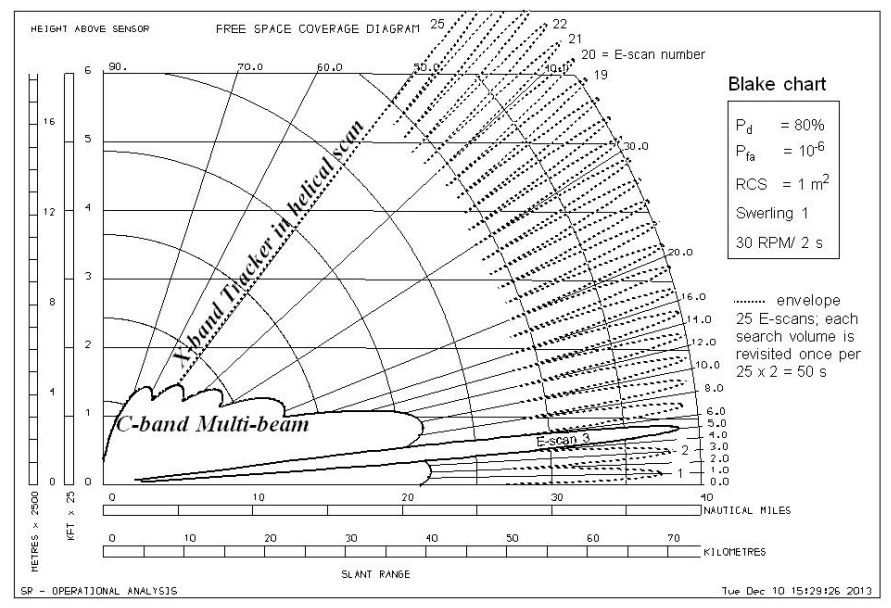

Fig. 4. $80 \%$ Free space Blake chart coverage diagram for 30 RPM rotating multi-beam surveillance and sequential scanned pencil beam tracker

Now, a straightforward example is elaborated by hand to reveal the difference in operational designation performance:

A sea skimming anti-ship missile threat approaches with $1000 \mathrm{~m} / \mathrm{s}$. Best-case the tracker's E-scan1 initially detects the threat at $70 \mathrm{~km}$ (worst-case $24 \times 2 \mathrm{~s}=48 \mathrm{~s}$ later at $70-$ $48=22 \mathrm{~km}$ ). Promotion to firm track costs 2 to 4 updates of E-scan 1 or 2 to $4 \times 25 \times 2 \mathrm{~s}=100$ to $200 \mathrm{~s}$ or 100 to 200 $\mathrm{km}$ later. Hence, firm track-while-scan is not established before the threat penetrates the radar platform. Multi-beam surveillance radar establishes firm track around $35 \mathrm{~km}$. So, straightforward inclusion of the firm track requirement shows the complete failure of the sequential scanned tracker as designator. The multi-beam provides in-time designation to effectively defend the radar platform against the modern agile multi-threat.

Calculation of the $\mathrm{CR}_{\mathrm{el}}$ value confirms the practical weakness of sequential scanned pencil beam as multi-threat designator, i.e. 65 vs. $720[\mathrm{~km} . \%$ s] for the multi-beam, while the Blake chart Fig. 4 appears to indicate the opposite. Radar selection based on the Blake chart-only would be a deadly mistake. The easy to compute CR metric helps to prevent this.

Besides the $\mathrm{CR}_{\mathrm{el}}$ in $[\mathrm{km} . \%$ s] used to quantify suitability for the anti-air warfare task, volume coverage rate $\mathrm{CR}_{\mathrm{V}}$ in $\left[\mathrm{km}^{3} / \mathrm{s}\right]$ is introduced in (2). This quantifies search radar suitability for situational awareness and air traffic control or surveillance.

\section{B. Coverage Information Rate to Qualify Awareness}

For surveillance, the quality of the target information in the coverage are of great importance for the reliability of the recognized picture. The digital photo image series at the bottom of Fig. 2 shows the quality improvement and better capability to classify and identify the detected capital character " $\mathbf{R}$ " going from low (1x1 pixel) to high resolution (100x100). Both the Blake sheet coverage and the CR metric fail to take into account the number of radar coordinates (2D, 3D, 4D) and their resolution, which are clearly discriminators for image quality. In other words, appreciation of the increased resolution capability of accurate modern 4D search radar is absent up to now. The CIR includes the number (here four, defined in section II.B) instantaneously unambiguous measured radar coordinates and their effective resolution.

In this section, search radar measurement principles are indicated in dimensions, i.e. 2D (RB), 3D (RBE) and 4D $\left(\mathrm{RBEV}_{\mathrm{rad}}\right)$; 2.5D means RBE-plots with poor angular resolution. The resolution is an indication of the measurement quality in terms of accuracy, resolution of closely spaced targets, discrimination from non-targets (natural, biological, man-made clutter) and additional classifying radar target features as provided by high range-Doppler resolution imaging. In other words: The more radar coordinates and resolution, the better the search radar supports automatic multi-dimensional tracking, classification and identification. So, enhanced target information and higher robustness under varying natural and electronic environments, i.e. with no/low degradation under increasingly stressing scenarios/conditions.

In order to illustrate the use of the CIR metric and radar evolution, an active phased array antenna is chosen. With this approach other antenna principles can be readily simulated by applicable antenna element weighting, i.e. going from the old fashioned rotating $2 \mathrm{D}$ fan beam to the most up-to-date modern fixed panel 4D multi-beam search radar systems. 
Relevant surveillance radar and requirement analysis data are:

Instrumented range $[5,250]^{8} \mathrm{~km}$; speed $[30,1500] \mathrm{m} / \mathrm{s}$. A $5 \mathrm{~s}$ update rate is required for the search volume up to $70^{\circ} ; 2^{\circ}$ pencil in azimuth. The rotating pencil beam reference radar $(\mathrm{H}$ $\mathrm{x} \mathrm{V}=2^{\circ} \times 6.4^{\circ}$ ) achieves $\mathrm{R}_{\mathrm{o}}=200 \mathrm{~km}$ as Blake chart result ${ }^{9}$.

\section{1) $2 D$ Pencil beam reference}

Remark that the $70^{\circ}$ requirement is not met.

The CIR is now quickly analyzed for the search radar evolution from 2D to 4D (results summary see Fig. 5):

\section{2) 2 D Fan beam cosec $^{2}$-pattern}

In order to widen the coverage of the single reference pencil beam a classical $\operatorname{cosec}^{2}$-pattern is employed: Up to $40^{\circ}$ instead of $70^{\circ}$ to prevent disproportional loss. This gives a moderate penalty in one-way loss of $2.5 \mathrm{~dB}$, thus two-way 5 $\mathrm{dB}$ : Blake chart degradation $\rightarrow \mathrm{R}_{\mathrm{o}}=150 \mathrm{~km}$.

\section{3) $2 D$ Fan beam with $P C$}

The next step is a higher bandwidth pulse modulation. Pulse compression (PC) provides an improved rangeresolution, -measurement accuracy and clutter discrimination: $\Delta \mathrm{R}=5000 \rightarrow 100 \mathrm{~m}$, resulting in higher quality information without a penalty in coverage.

\section{4) 2 D Fan beam with MTI}

Adding moving target indication or MTI-filtering discriminates moving targets from non- and slowly-moving clutter. Resolution of air- and surface-targets in the MTI/nonMTI-channel. MTI-loss is $2 \mathrm{~dB} \rightarrow \mathrm{R}_{\mathrm{o}}=134 \mathrm{~km}$.

\section{5) 2.5D Sequential Scanned Pencil Beam (see Fig. 2b))}

Electronic beam scheduling manages the dwell time and $R_{o}$ per elevation position (eleven beam positions to cover $70^{\circ}$ ) during the time-on-target to create a $\operatorname{cosec}^{2}$-pattern: $\mathrm{R}_{\mathrm{o}}=150$ $\mathrm{km}$. Beam-agility limits the MTI application in the upper beams (short dwell times), i.e. at two lowest beams at horizon only $\left(\mathrm{MTI}_{\mathrm{HS}}\right)$ and a relatively poor angular accuracy $\rightarrow 2.5 \mathrm{D}$.

\section{6) 3D Four Face Fixed Passive Phased Array}

In the 1970-s the multi-function radar has been introduced. "Passive" means that the transmitter energy is equally distributed over the faces. Beam scheduling is the same as above. Extra loss is $2 \mathrm{~dB} \rightarrow \mathrm{R}_{\mathrm{o}}=134 \mathrm{~km}$ and resolution degrades $^{10}$, which is compensated by mono-pulse techniques. The latter is introduced in the multi-function radar to provide angular accuracies as required for fire control.

\section{7) 3D Four Face Fixed Active Phased Array}

Active antennae allow simultaneous transmission resulting in four times longer time-on-target per sector $\mathrm{R}_{\mathrm{o}}=189 \mathrm{~km}$.

\footnotetext{
${ }^{8}$ Low resolution and low PRF assumed, i.e. uncompressed pulse $5 \mathrm{~km}$ (i.e. $331 / 3 \mu$ s pulse with $0.03 \mathrm{MHz}$ bandwidth) and PRF is lower than $600 \mathrm{~Hz}$ (250 km unambiguous range) ${ }^{9}$ Result computed at broadside antenna is assumed for the target of interest (e.g. $1 \mathrm{~m}^{2}$ SW1) and incoherent pulse integration

${ }^{10}$ Due to off-broadside scan loss and beam broadening effect
}

\section{8) 4D Multi-beam (see Fig. 2c))}

Multi-beam rotator conserves time-on-target at the cost of $2.3 \mathrm{~dB} \operatorname{cosec}^{2}$-taper loss on transmit. Long time-on-target allows medium PRF regimes providing unambiguous range and radial velocity resolution at the cost of $3 \mathrm{~dB} \rightarrow \mathrm{R}_{\mathrm{o}}=147$ $\mathrm{km}$.

9) 4D Multi-beam Four Face Fixed Active Phased Array

2D Multi-beam gives better angular and much better Doppler resolution (8x longer time-on-target) $\rightarrow R_{o}=165 \mathrm{~km}$.

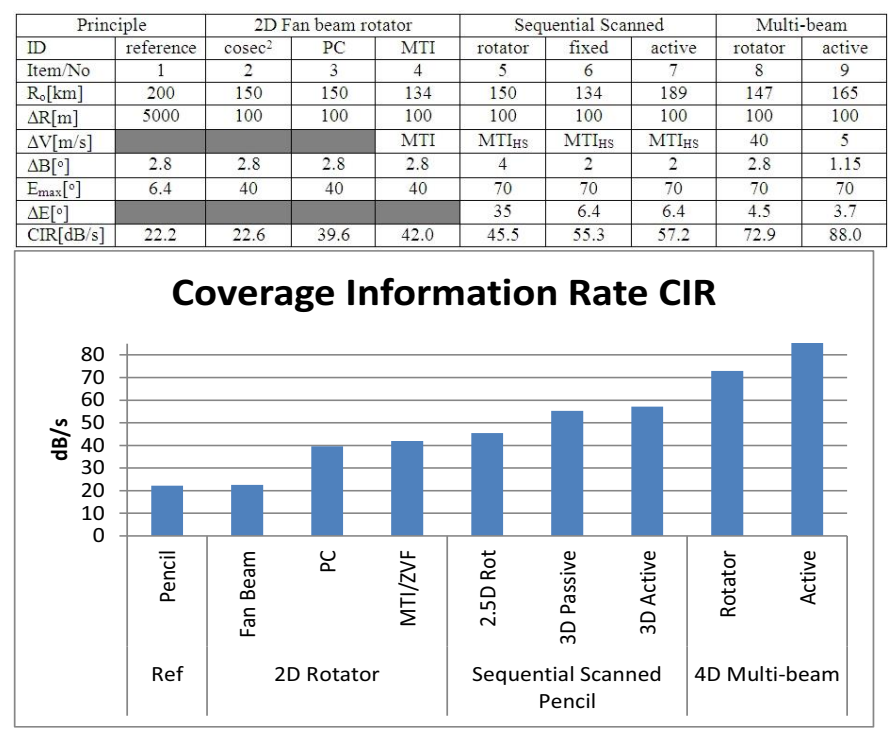

Fig. 5. Coverage information rate summary conventional 2D fan beam up to modern 4D Multi-beam four face fixed active phased array surveillance radar

\section{CONCLUSION}

Alternative coverage metrics have been introduced to qualify different search radar candidates for different operational tasks. To illustrate the ease of computation starting with a reference radar system- the evolution from $2 \mathrm{D}$ fan beam up to the most modern 4D four face phased array multi-beam search radar have been analyzed. Blake's coverage has been extended with three coverage rate metrics, which indicate the suitability for anti-air warfare $\left(\mathrm{CR}_{\mathrm{el}}\right.$ for in time designation of missile threat between $\left.0-70^{\circ}\right)$, air surveillance or traffic control $\left(\mathrm{CR}_{\mathrm{V}}\right)$ and situational awareness (CIR).

As shown graphically in the bar chart in Fig. 5 the evolution from 2D to $4 \mathrm{D}$ search radar tends to enhance the CIR. The latter principle appears to be more appropriate to serve critical and situational awareness aspects by providing a high 4D information quality and operational robustness (low false alarm-, false track- and false classification-rate).

\section{REFERENCES}

[1] L.V. Blake, "A Guide to Basic Pulse-Radar Maximum Range Calculation”, NRL report 5868, 1962 